\title{
Nanocrosses with Highly Tunable Double Resonances for Near-Infrared Surface-Enhanced Raman Scattering
}

\author{
Ye Jian ${ }^{1,2}$ and VanDorpe Pol ${ }^{1}$ \\ ${ }^{1}$ IMEC vzw, Kapeldreef 75, 3001 Leuven, Belgium \\ ${ }^{2}$ Chemistry Department, KU Leuven, 3001 Leuven, Belgium \\ Correspondence should be addressed to Ye Jian, jian.ye@imec.be
}

Received 14 October 2011; Accepted 2 December 2011

Academic Editor: A. Femius Koenderink

Copyright ( 2012 Y. Jian and V. Pol. This is an open access article distributed under the Creative Commons Attribution License, which permits unrestricted use, distribution, and reproduction in any medium, provided the original work is properly cited.

\begin{abstract}
We present asymmetric gold nanocrosses with highly tunable double resonances for the near-infrared (NIR) surface-enhanced Raman scattering (SERS), optimizing electric field enhancement at both the excitation and Stokes Raman wavelengths. The calculated largest SERS enhancement factor can reach a value as large as $1.0 \times 10^{10}$. We have found that the peak separation, the resonance position, and peak intensity ratio of the double-resonance gold nanocrosses can be tuned by changing the structural dimensions or the light polarization.
\end{abstract}

Surface-enhanced Raman scattering (SERS) effect boosts the Raman signals of adsorbates on the surfaces of metallic nanostructures when their plasmon resonances and consequently an enhanced near field are excited at the wavelength of the stimulating laser beam. The enhancement factor (EF) of SERS may reach a level of single-molecule resolution $[1,2]$. The SERS technique has demonstrated its use in many applications such as a $\mathrm{pH}$ meter, biosensing, and bioimaging [3-5]. Recently, because of the usage of SERS for in vivo bioapplications, there is increased interest of SERS in the near-infrared (NIR) region (700-1400 nm) in the optical spectrum, where blood and tissue are most transparent and light can penetrate tissue over a deepest distance $[6,7]$.

There are two mechanisms behind SERS, electromagnetic (EM) and charge transfer (CT) effect, which are extensively mentioned in the literature. It is, however, generally accepted that the EM effect is the dominant one. In the EM mechanism, the EF of each adsorbate is proportional to the product of the field intensity at the laser excitation wavelength and that at the Stokes-shifted Raman scattering wavelength, namely, $\mathrm{EF}=\left|\mathrm{EF}_{\mathrm{ex}}\right|^{2}\left|\mathrm{EF}_{\text {scat }}\right|^{2}$. As plasmon resonances, which are usually responsible for the EM enhancement mechanism, are spectrally relatively broad, usually it is assumed that $\left|\mathrm{EF}_{\mathrm{ex}}\right|^{2}$ and $\left|\mathrm{EF}_{\text {scat }}\right|^{2}$ are equal if the center of the resonance is chosen in between the two wavelength positions. For NIR SERS, however, the Raman scattering wavelength and the excitation wavelength can be separated by over $100 \mathrm{~nm}$, limiting the efficiency of a single resonance. Very recently, SERS substrates with double plasmon resonances at the laser excitation and Raman scattering wavelengths have been demonstrated by Crozier's group by showing higher enhancement compared to similar structures with single resonances $[8,9]$. In addition, it is well known that a strong plasmonic coupling in the structures (e.g., bow-tie and disk dimer) will generate the near-field hot spots in the tiny gap, which may amplify the Raman signal significantly [10-13]. In this paper, we report on a different type of double-resonance plasmonic substrate consisting of gold $(\mathrm{Au})$ nanocrosses potentially useful for the NIR SERS application. Strong near field enhancement is achieved by the plasmonic coupling between two opposite tips of the crossbars. The achievement of the double resonances can be realized by breaking the symmetry of the Au nanocross. We have found that the peak separation (namely, the distance between the excitation and scattering wavelength), the resonance position, and peak intensity ratio of the double-resonance $\mathrm{Au}$ nanocrosses are highly tunable in the NIR range by changing the light polarization or the structural dimensions. We have also numerically demonstrated that the SERS EF of the Au nanocross can reach a value as large as $1.0 \times 10^{10}$. 


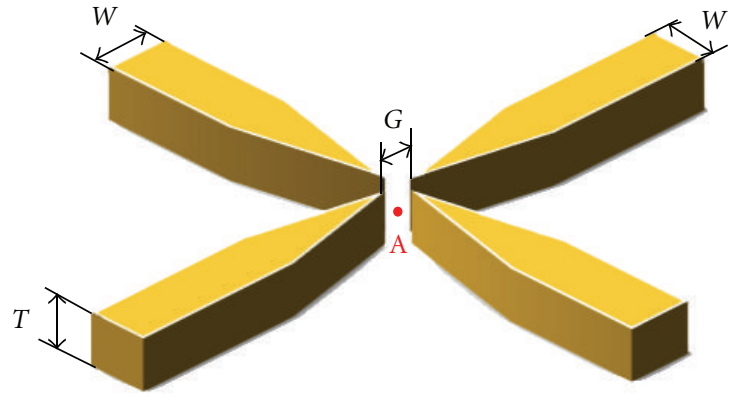

(a)

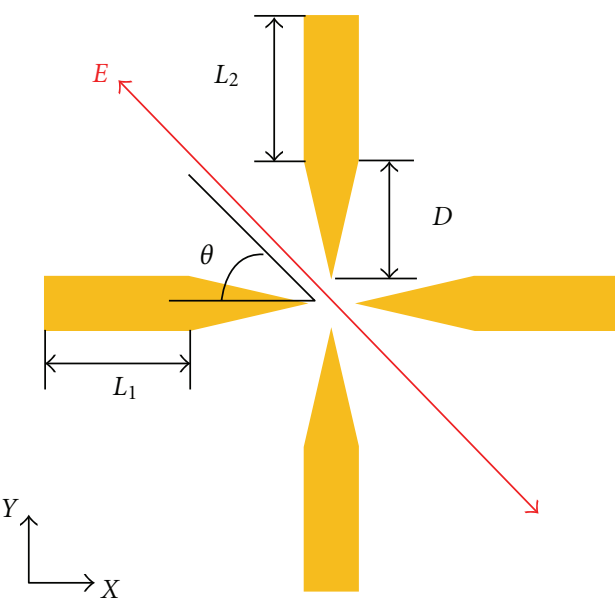

(b)

Figure 1: Schematic and dimensional parameters of an Au nanocross. The symmetry of the Au nanocrosses can be broken by tuning the different arm lengths of $L_{1}$ and $L_{2}$.
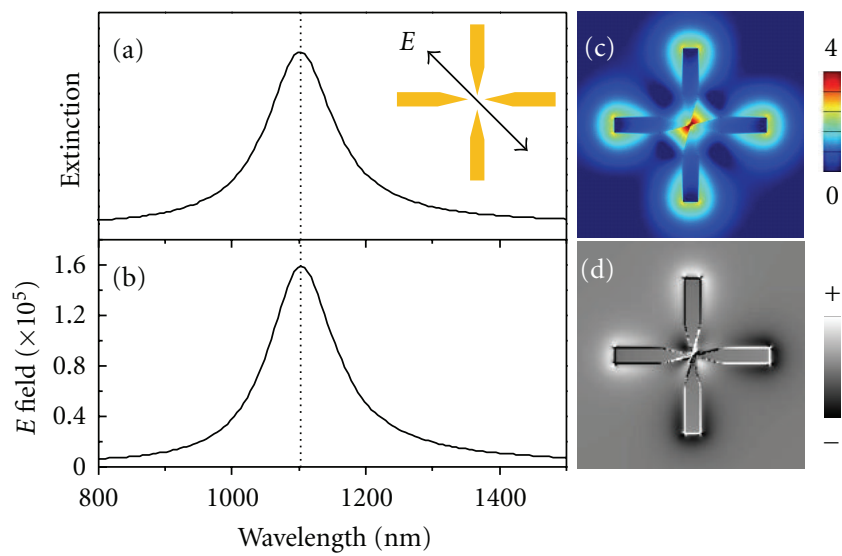

Figure 2: (a) Extinction spectrum, (b) electric field intensity $\left(\left|E^{2}\right|\right)$ spectrum at the point A (see Figure 1), (c) the on-resonance electric field distribution $\left(\log \left|E^{2}\right|\right)$, and (d) surface charge distribution for a symmetric Au nanocross $(120,120)$ normally illuminated by a $\theta=$ $45^{\circ}$ polarized plane wave.

We employed the three-dimensional finite different time domain (FDTD) calculation method to investigate the optical properties of $\mathrm{Au}$ nanocrosses in this work. Figures 1(a) and 1(b) display the schematic illustration and dimensional parameters of the Au nanocross used in the simulation with a width $(W)$, thickness $(T)$, gap size $(G)$, tip length $(D)$, arm length along $X$ axis $\left(L_{1}\right)$, and arm length along $Y$ axis $\left(L_{2}\right)$. Herein, an Au nanocross with $L_{1}$ and $L_{2}$ can be denoted $\left(L_{1}\right.$, $L_{2}$ ) because the other dimensional parameters are constant ( $W=40 \mathrm{~nm}, T=40 \mathrm{~nm}, G=4 \mathrm{~nm}$, and $D=78 \mathrm{~nm}$ ). $L_{1}$ and $L_{2}$ are varied to achieve the double resonances at different wavelengths and with different peak separations. The light is propagating normal to the $X Y$ plane and is polarized along the direction of $\theta=45^{\circ}$ (see Figure 1(b)). The simulations were performed by using the FDTD software from Lumerical Solutions, Inc. The empirical dielectric function of $\mathrm{Au}$ has been described in our previous work [14]. We have placed
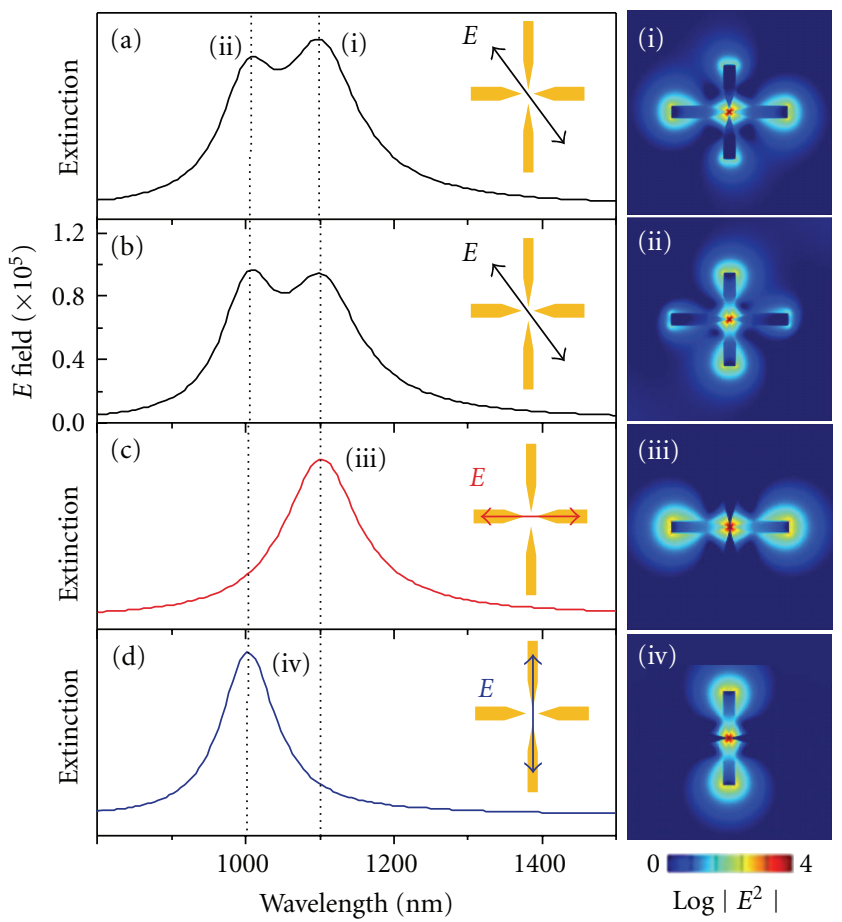

Figure 3: (a) Extinction spectrum and (b) electric field intensity $\left(\left|E^{2}\right|\right)$ spectrum at the point A (see Figure 1 ) for an asymmetric $\mathrm{Au}$ nanocross $(120,80)$ normally illuminated by a $\theta=45^{\circ}$ polarized plane wave. (c, d) Extinction spectra of the Au nanocross $(120,80)$ with a polarization along the (c) $x$ - and (d) $y$-axis. The electric field distributions $\left(\log \left|E^{2}\right|\right)$ of the plasmon resonance modes (i-iv) are shown on the right.

a point monitor (point A) in the middle of the structure gap, whose position is indicated in Figure 1(a). All extinction spectra, electric field intensity spectra at the point A, electric field distribution, and surface charge distribution images were obtained from the simulations. The amplitude of the electric field of the incident light is set to 1 . 


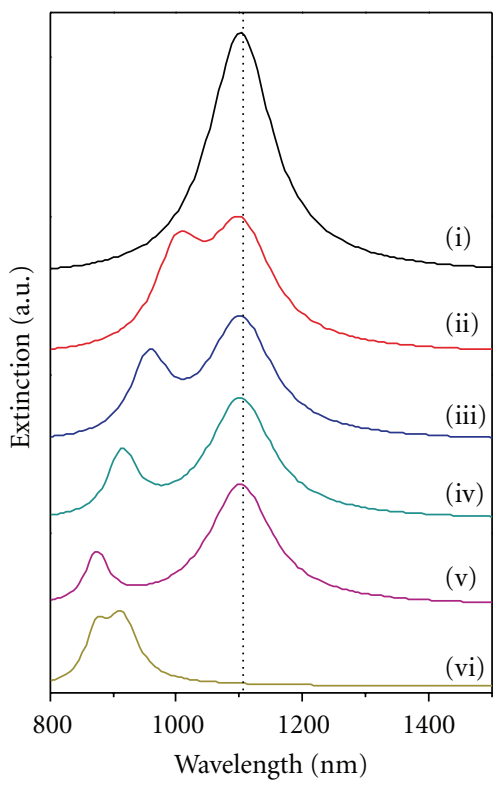

(a)

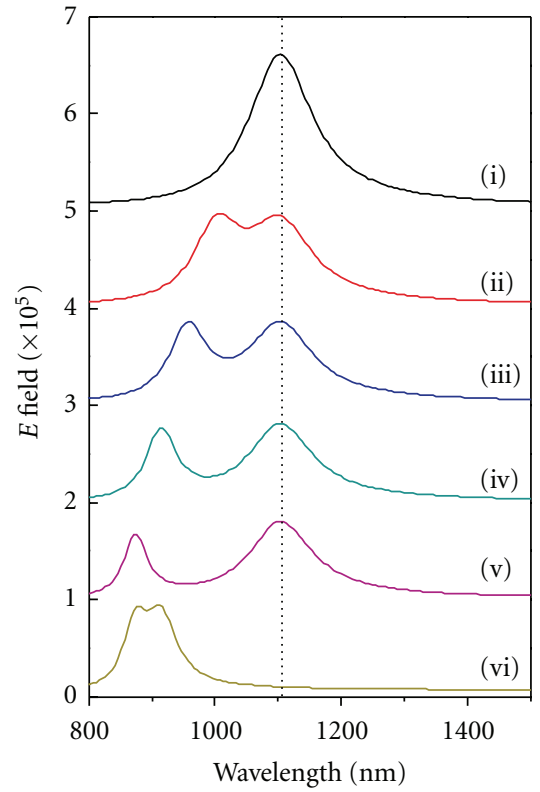

(b)

FIgURE 4: (a) Extinction and (b) electric field intensity $\left(\left|E^{2}\right|\right)$ spectra at the point A (see Figure 1) for different dimensional Au nanocrosses: (i) 120, 120; (ii) 120, 80; (iii) 120, 60; (iv) 120, 40; (v) 120, 20; and (vi) 40, 20.

Au nanocrosses may strongly couple to the incident radiation by the dipolar mode and higher order modes, but the higher order modes are dark for the normal incidence [15]. For a symmetric Au nanocross $(120,120)$ normally illuminated by $\theta=45^{\circ}$ polarized light, we can only observe a bright resonance mode at $1103 \mathrm{~nm}$ in the NIR range (Figure 2(a)). In order to fully understand its mode feature, we plot its electric field distribution image in Figure 2(c). It is clearly shown that each arm of the Au nanocross displays a strong dipolar resonant character and couples to each other, consequently producing a rather enhanced electric field in the gap. The electric field intensity spectrum at the point $\mathrm{A}$ shows that the maximum electric field $\left(|E|^{2}\right)$ can reach to a value as large as $1.6 \times 10^{5}$. The calculated charge distribution image shown in Figure 2(d) further confirms the dipolar nature of the modes and their strong coupling for the arms of the Au nanocross.

To achieve the double resonances for SERS, we break the symmetry of the Au nanocross by changing the arm length $L_{2}$. Figure $3($ a) shows an extinction spectrum of an asymmetric Au nanocross $(120,80)$ with a decreased value of $L_{2}$. We can observe two resonance at 1103 (i) and $1007 \mathrm{~nm}$ (ii). Interestingly, the corresponding electric field intensity spectrum at the point $\mathrm{A}$ shows two maximum values at the same two resonance positions, which can be used for the SERS excitation and the scattering enhancement. More importantly, the electric field intensity spectrum shows that the field enhancements of these two resonances both reach a value of around $1.0 \times 10^{5}$ (Figure $3(\mathrm{~b})$ ). Therefore, the total SERS EF may reach a value of $1.0 \times 10^{10}$. We simulated the excitation spectra of the Au nanocross $(120,80)$ with a light polarization along the long arm ( $x$-axis) and the short arm ( $y$-axis) in Figures 3(c) and 3(d) in order to determine the origin of the prescribed two resonances' (i) and (ii) formation. It is shown that the resonance (i) and (ii) are actually from the resonance (iii) and (iv), formed by the light polarization along the long and short arms, respectively. The electric field distributions of the resonances mode in Figure 3 further show that the dominant modes of resonance (i) and (ii) are rather similar to that of (iii) and (iv), respectively, which indicates a weak coupling between two bright modes (iii) and (iv) resulting in the double-resonance feature. Figure 3 also implies that we can easily tune the resonance positions by controlling the arm lengths of the Au nanocrosses. This is very common to tune the resonance position by controlling the asymmetric geometry of the plasmonic structure, for example, asymmetric split ring resonators $[16,17]$.

In Figure 4(a), we show the excitation and the electric field intensity spectra of six different dimensional Au nanocrosses: (i) 120,120; (ii) 120, 80; (iii) 120, 60; (iv) 120, 40; (v) 120,20 ; and (vi) 40, 20. Some plasmonic resonant behaviors are observed: (1) two resonances are highly tunable in the NIR range; (2) the resonance at higher wavelength corresponds to the mode along the long arm of the nanocross, and the lower resonance to that of the short arm; (3) the more difference between the long and short arm, the larger the peak separation; (4) for different dimensional nanocrosses, the field enhancement for both resonances can reach a value in the range of $5.0 \times 10^{4}-1.0 \times 10^{5}$; consequently, the total SERS EF remaining in the range of $1.0 \times$ $10^{9}-1.0 \times 10^{10}$. For the structures of (ii), (iii), (iv) and (v), one resonance remains at $1103 \mathrm{~nm}$ and the other one can be tuned from 1007 to $872 \mathrm{~nm}$ by changing the short arm from 


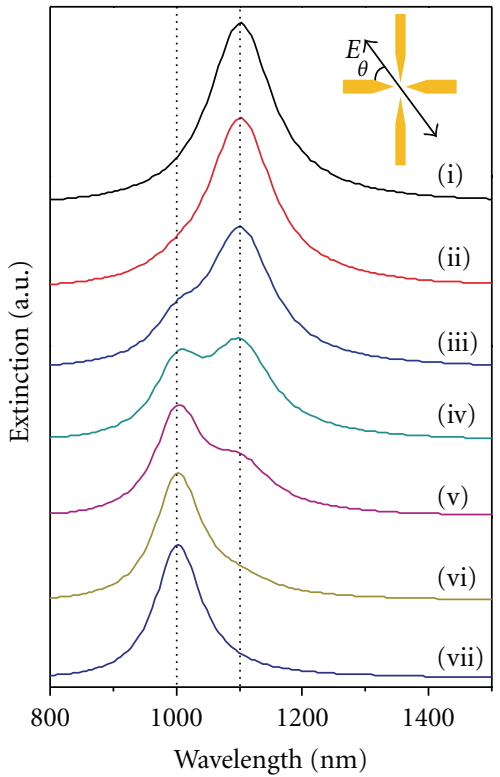

(a)

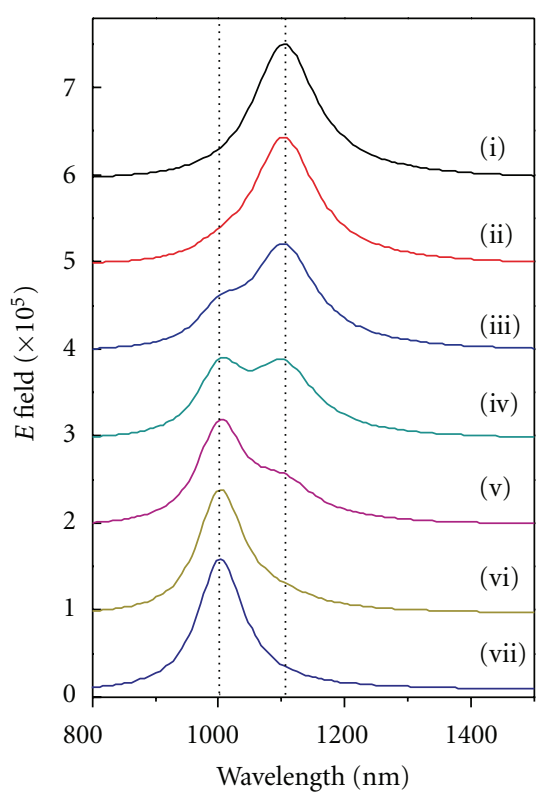

(b)

Figure 5: (a) Extinction and (b) electric field intensity $\left(\left|E^{2}\right|\right)$ spectra at the point A (see Figure 1) for a Au nanocross (120, 80) illuminated by a plane wave with different polarizations. The angle $(\theta)$ between the light polarization and the $x$-axis is (i) 0 , (ii) 15 , (iii) 30 , (iv) 45 , (v) 60, (vi) 75, and (vii) 90 degree.

80 to $20 \mathrm{~nm}$. The peak separation can be controlled from 30 to $231 \mathrm{~nm}$ (Figure 4(b)). The high tunability and flexibility of the double-resonance position and peak separation offer us a couple of advantages for SERS. First, we can easily match the double resonances of the $\mathrm{Au}$ nanocross to the laser line and the Stoke Raman band. Second, the large peak separation allows us to enhance the Raman band, for example, $v(=\mathrm{CH})$, having a Stoke Raman shift at a longer wavenumber at around $3000 \mathrm{~cm}^{-1}$, which is $240 \mathrm{~nm}$ away from the NIR laser line (e.g., $785 \mathrm{~nm}$ ). In addition, we believe that the double-resonance nanocross is potential useful for the surface-enhanced second harmonic generation [18]. The plasmon resonances of the asymmetric Au nanocrosses are not only arm length dependent but also incident light polarization dependent. Figures 5(a) and 5(b) show the extinction and electric field intensity spectra of the $\mathrm{Au}$ nanocross $(120,80)$ with different light polarizations. By changing the polarization angle $(\theta)$, we can control the peak intensity ratio between the two resonances of the $\mathrm{Au}$ nanocross at 1007 and $1103 \mathrm{~nm}$, respectively. Moreover, we have simulated the double-resonance nanocross with rounded tips (with a $4 \mathrm{~nm}$ curvature radius) in order to have a more realistic estimation for the experimental samples. We have found that the maximum SERS EF drops to $2.0 \times$ $10^{9}$ for the Au nanocross $(120,80)$, one order of magnitude smaller than that with sharp tips, which is still a rather large $\mathrm{EF}$ for the molecular detection.

In conclusion, we have numerically demonstrated asymmetric Au nanocrosses as double-resonance SERS substrates. Strong field enhancements at the excitation and scattering wavelengths lead to a total SRS EF of around $1.0 \times 10^{10}$. Moreover, we have shown that the resonance position, the peak separation, and the peak intensity ratio of the $\mathrm{Au}$ nanocrosses can be tuned by changing their arm lengths and the light polarization. Strong near field and large peak separation are particularly important to the enhancement of the Raman band at high wavenumbers. We anticipate that this work will offer an aid for the design and the fabrication of new SERS substrates with a large EF.

\section{Acknowledgment}

J. Ye and P. V. Dorpe acknowledge financial support from the FWO of Flanders.

\section{References}

[1] K. Kneipp, Y. Wang, H. Kneipp et al., "Single molecule detection using surface-enhanced Raman scattering (SERS)," Physical Review Letters, vol. 78, no. 9, pp. 1667-1670, 1997.

[2] S. Nie and S. R. Emory, "Probing single molecules and single nanoparticles by surface-enhanced Raman scattering," Science, vol. 275, no. 5303, pp. 1102-1106, 1997.

[3] S. W. Bishnoi, C. J. Rozell, C. S. Levin et al., "All-optical nanoscale pH meter," Nano Letters, vol. 6, no. 8, pp. 1687-1692, 2006.

[4] D. Graham, K. Faulds, and W. E. Smith, "Biosensing using silver nanoparticles and surface enhanced resonance Raman scattering," Chemical Communications, no. 42, pp. 4363-4371, 2006.

[5] Y. Huang, V. P. Swarup, and S. W. Bishnoi, "Rapid raman imaging of stable, functionalized nanoshells in mammalian cell cultures," Nano Letters, vol. 9, no. 8, pp. 2914-2920, 2009.

[6] R. Weissleder, "A clearer vision for in vivo imaging," Nature Biotechnology, vol. 19, no. 4, pp. 316-317, 2001. 
[7] A. M. Smith, M. C. Mancini, and S. Nie, "Bioimaging: second window for in vivo imaging," Nature Nanotechnology, vol. 4, no. 11, pp. 710-711, 2009.

[8] Y. Chu, M. G. Banaee, and K. B. Crozier, "Double-resonance plasmon substrates for surface-enhanced raman scattering with enhancement at excitation and stokes frequencies," ACS Nano, vol. 4, no. 5, pp. 2804-2810, 2010.

[9] M. G. Banaee and K. B. Crozier, "Mixed dimer double-resonance substrates for surface-enhanced Raman spectroscopy," ACS Nano, vol. 5, no. 1, pp. 307-314, 2011.

[10] Z. Zhang, A. Weber-Bargioni, S. W. Wu, S. Dhuey, S. Cabrini, and P. J. Schuck, "Manipulating nanoscale light fields with the asymmetric bowtie nano-colorsorter," Nano Letters, vol. 9, no. 12, pp. 4505-4509, 2009.

[11] I. Romero, J. Aizpurua, G. W. Bryant, and F. J. García De Abajo, "Plasmons in nearly touching metallic nanoparticles: singular response in the limit of touching dimers," Optics Express, vol. 14, no. 21, pp. 9988-9999, 2006.

[12] M. I. Stockman, L. N. Pandey, L. S. Muratov, and T. F. George, "Comment on "photon scanning tunneling microscopy images of optical excitations of fractal metal colloid clusters"', Physical Review Letters, vol. 75, no. 12, p. 2450, 1995.

[13] N. J. Halas, S. Lal, W.-S. Chang, S. Link, and P. Nordlander, "Plasmons in strongly coupled metallic nanostructures," Chemical Reviews, vol. 111, no. 6, pp. 3913-3961, 2011.

[14] J. Ye, N. Verellen, W. Van Roy et al., "Plasmonic modes of metallic semishells in a polymer film," ACS Nano, vol. 4, no. 3, pp. 1457-1464, 2010.

[15] N. Verellen, P. Van Dorpe, C. Huang et al., "Plasmon line shaping using nanocrosses for high sensitivity localized surface plasmon resonance sensing," Nano Letters, vol. 11, no. 2, pp. 391-397, 2011.

[16] B. Lahiri, A. Z. Khokhar, R. M. De La Rue, S. G. Mcmeekin, and N. P. Johnson, "Asymmetric split ring resonators for optical sensing of organic materials," Optics Express, vol. 17, no. 2, pp. 1107-1115, 2009.

[17] V. A. Fedotov, M. Rose, S. L. Prosvirnin, N. Papasimakis, and N. I. Zheludev, "Sharp trapped-mode resonances in planar metamaterials with a broken structural symmetry," Physical Review Letters, vol. 99, no. 14, Article ID 147401, 2007.

[18] N. J. Borys, M. J. Walter, and J. M. Lupton, "Intermittency in second-harmonic radiation from plasmonic hot spots on rough silver films," Physical Review B, vol. 80, no. 16, Article ID 161407, 2009. 

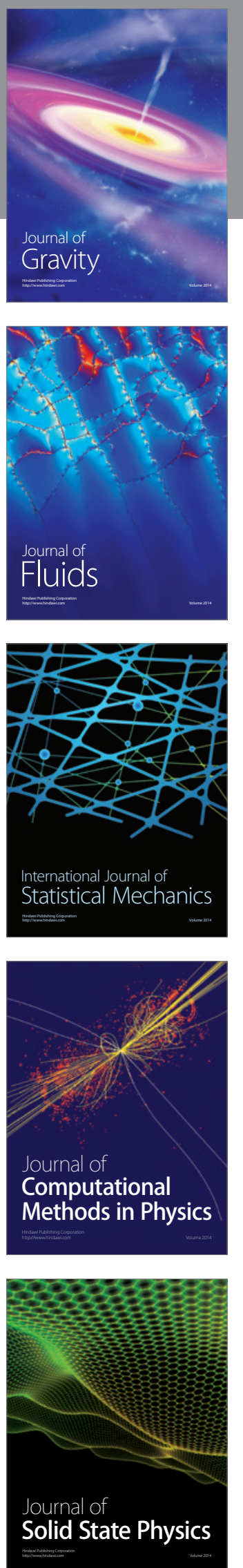

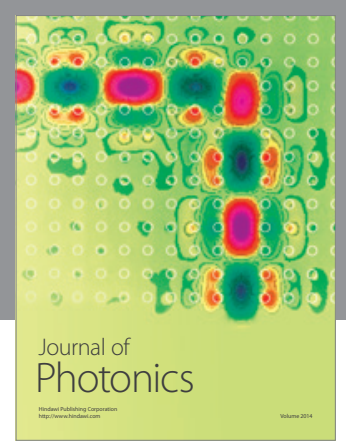

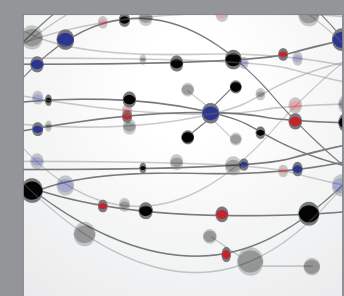

The Scientific World Journal
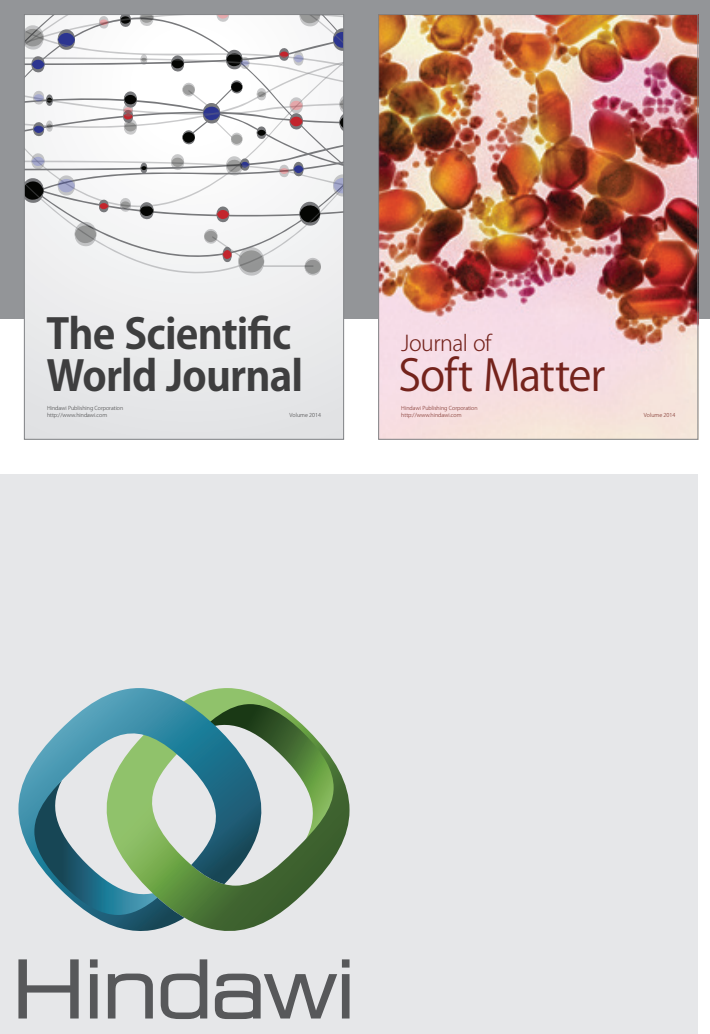

Submit your manuscripts at

http://www.hindawi.com
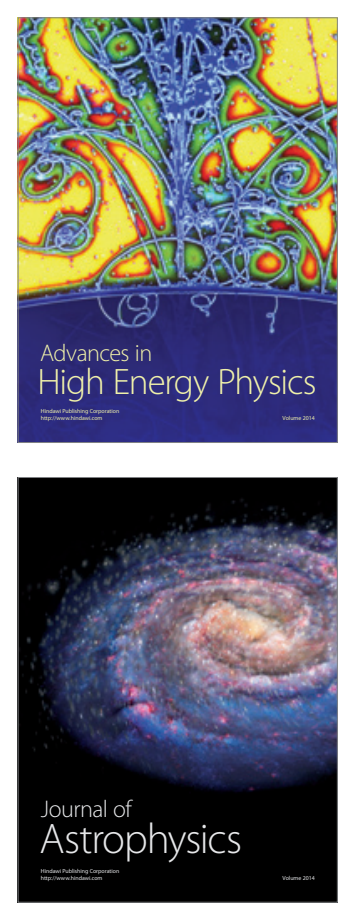
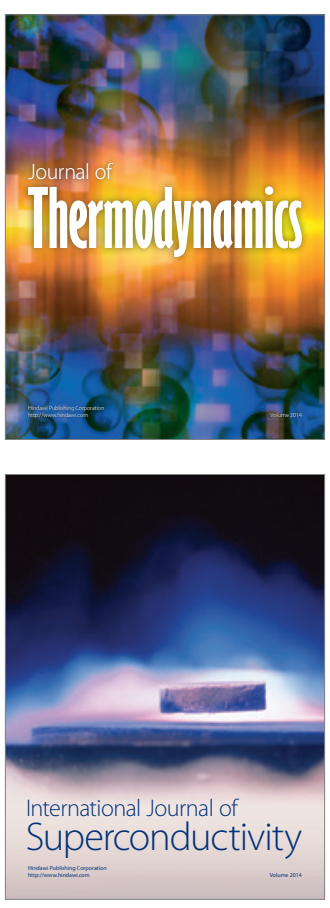
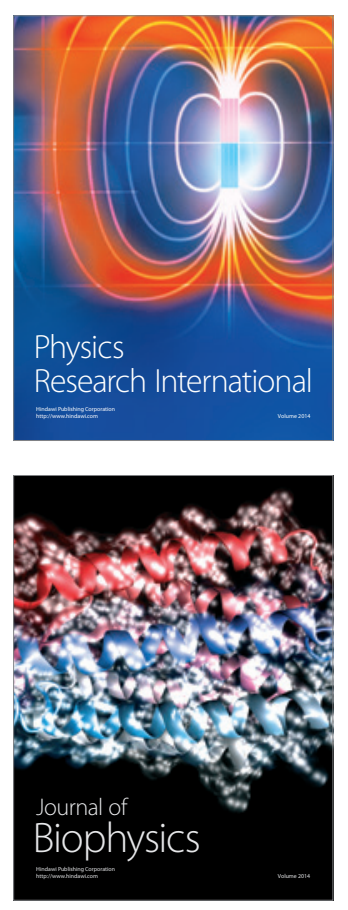
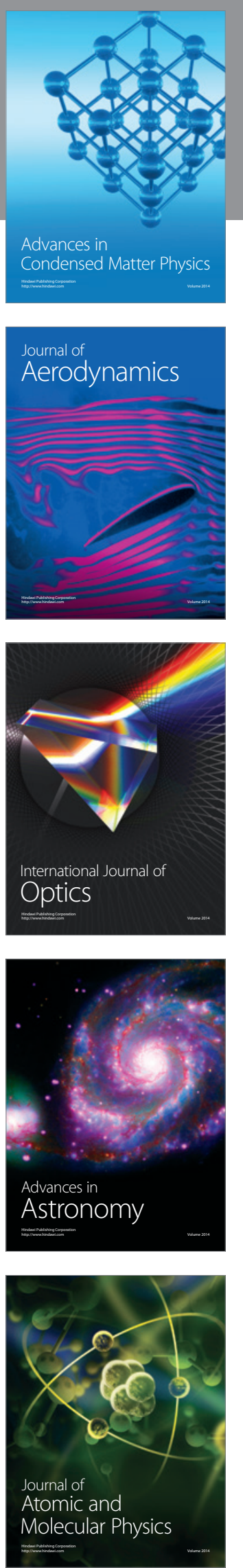